\title{
Innovations for future systems
}

\author{
Haroon Malik ${ }^{1}$ Ansar-Ul-Haque Yasar $^{2}$ - Elhadi M. Shakshuki ${ }^{3}$
}

Published online: 28 June 2017

(c) Springer-Verlag GmbH Germany 2017

This special issue is based on the best papers from the 11th International Conference on Future Networks and Communications (FNC), collocated with The 13th International Conference on Mobile Systems and Pervasive Computing, which were held in Montreal, Quebec, Canada on 15-18 August 2016. Both conferences attracted a large number of scientific papers that contributed to the state-of-theart in the areas of mobile computing and impending networks. All the papers selected for this special issue has been extended from their original versions and underwent two rounds of rigorous peer-review process. The accepted papers are based on the reviewers' feedback, as well as the evaluations of the Guest Editors, seven papers were selected for this special issue. The accepted papers cover forthcoming development and emerging research along mobile computing and connected systems topics such as ultra-large scale software systems, cloud computing environments, development of routing algorithms, resource allocation in optical grids and stream mining.

The first paper by Mahmoud Al-Ayyoub is entitled "An Extended Study of Collaboration Networks of Levantine

Elhadi M. Shakshuki

elhadi.shakshuki@acadiau.ca

Haroon Malik

malikh@marshall.edu

Ansar-Ul-Haque Yasar

ansar.yasar@uhasselt.be

1 Weisberg Division of Computer Science, Marshall University, Huntington, WV, USA

2 Transportation Research Institute, Hasselt University, Hasselt, Belgium

3 Jodrey School of Computer Science, Acadia University, Wolfville, Canada
Biomedical Researchers". A collaboration (or a co-authorship) network (ColNet) is a type of $\mathrm{SN}$ in which the actors are scholars/researchers and a tie between two actors represents the fact they have co-authored at least one paper together. Scholars have been analyzing ColNet extensively to recognize affected factors in behavior, motivation and performance of collaborations. The paper analyzed ColNet for levantine researchers in the biomedical field. The analysis is focused on the patterns of scientific collaboration based on the published papers found on PupMed from 1991 till 2010 years. Analyzing the ColNet reveals that local researchers are shifting from working individually or with non-local collaborators to working within large groups of local collaborators relatively.

The second paper by K. Vidyasankar is entitled "Atomicity of Batches in Stream Processing". Stream processing is about processing continuous streams of data by the programs in a workflow. Continuous execution is discretized by grouping input stream tuples into batches and using one batch at a time for the execution of programs. The paper first defines the concept of atomic batches and later study the issues that hinder the atomicity of the batches when processed in the workflow simultaneously.

The third paper by Nadine Kashmar and Mirna Atieh is entitled "Mining Frequent Patterns to Identify Vertical Handover Parameters in Cellular Networks". The paper proposes a new approach to prove the most effective parameters for vertical hang over (VHO) process in cellular networks. The input parameters are very important to determine the most suitable VHO algorithm to reduce network signaling, decrease mobile device power loss and improve network quality of service. Towards this end, the paper presents two new algorithms: (a) DaSA algorithm, which is used to summarize the preprocessed data and find patters and (b) FPCA algorithm to find the frequency pattern of 
the simultaneous occurrence of the given parameters. The approach is evaluated on real world data from servers of two mobile telecom companies in Lebanon.

The fourth paper by Haroon Malik and Elhadi M. Shakshuki is entitled "Performance Evaluation of Counter Selection Techniques to Detect Discontinuity in LargeScale-Systems". One of the fundamental problems faced by analysts in preparing data for use in forecasting is the timely identification of data discontinuities. A discontinuity is an abrupt change in a time-series pattern of a performance counter that persists but does not recur. The paper (a) proposed supervised and an unsupervised techniques to automatically identify the important performance counters that are likely indicators of discontinuities within performance data and (b) compared the performance of the approaches by conducting a case study on the performance data obtained from a large scale cloud provider as well as on open source benchmarks systems.

The fifth paper by Gabriel Orsini et al. is entitled "Generic Context Adaptation for Mobile Cloud Computing Environments". The paper pitches the concept of the generic context adaptation (GCA) process that is able to predict arbitrary context attributes to anticipate the quickly changing context of mobile applications. A simulation of the developed GCA process that is based on realistic context information provided by the Nokia Mobile Data Challenge (MDC) campaign is presented. Moreover, the paper provides an evaluation of the GCA process in a Mobile Cloud Computing (MCC) offloading scenario for image processing.

The sixth paper by Saja Al Mamoori et al. is entitled "Energy-efficient Anycast Scheduling and Resource Allocation in Optical Grids". Due to rapid increase in the highbandwidth applications, the power consumption of communications equipment for such networks has been increasing steadily over the past decade. Therefore, energy efficient routing schemes and traffic models needs to be developed to reduce the energy consumption. The paper presents a new approach for energy aware routing and wavelength assignment (RWA), which jointly schedules demands (in time) and performs routing of sliding scheduled lightpath demands in optical grid networks by exploiting the flexibility of anycast principle. The approach implements a comprehensive energy-aware resource allocation for optical grid networks, which is able to consider power consumption over a wide variety of network components.

The seventh paper by Alaa E. Abdallah is entitled "Low Overhead Hybrid Geographic-based Routing Algorithms with Smart Partial Flooding for 3D Ad Hoc Networks". In flooding-based routing, when a packet reaches a node, it forwards that packet to all its neighbors. This can usually find the shortest path between two nodes. However, it can be difficult for these routing algorithms to work with large MANETs because of the huge traffic and overhead that can be created around the network. Moreover, the flooding algorithm usually address the routing environments in 2D space. However, in real life, nodes can be located in 3D space. The paper proposes 3D geographical routing algorithms that uses the advantage of the high delivery rate of the flooding-based algorithms and the low overhead of the progress-based routing algorithms. The paper evaluates the proposed algorithms using simulations. All the algorithms have excellent delivery rate and low overhead.

The guest editors would like to take this opportunity to thank all the authors for the efforts they put in the preparation of their manuscripts and for their valuable contributions. We wish to express our deepest gratitude to the referees who provided very useful and thoughtful feedback to our authors. Our sincere thanks go to the Editor-in-Chief for his kind help and support. 